\title{
Insatisfacción corporal y trastornos de conducta alimentaria en gimnastas: revisión sistemática \\ Body dissatisfaction and eating disorders in gymnasts: a systematic review
}

*,***Alicia Salas-Morillas, **Águeda Gutiérrez-Sánchez, *Mercedes Vernetta-Santana

*Universidad de Granada (España), **Universidad de Vigo (España), ***Instituto Universitario Alicia Alonso-Universidad Rey Juan Carlos (España)

Resumen. Introducción: En los deportes gimnásticos ciertas medidas morfológicas como el peso, un índice de masa corporal (IMC) y un porcentaje de grasa bajo adquieren importancia para el éxito deportivo. La preocupación excesiva por estos factores o alteración de la Imagen Corporal (IC), puede llevar a estos gimnastas a padecer Trastornos de Conducta Alimentaria (TCA). Objetivo: El propósito de este estudio fue analizar losTCA y la IC en practicantes de deportes gimnásticos mediante una revisión sistemática. Material y método: Siguiendo la lista de verificación «PRISMA» se realizó la búsqueda en cinco bases de datos electrónicas (WOS, PubMed, SPORTDiscus, Scopus y Google Scholar), de estudios transversales en inglés, español y portugués publicados desde enero del 2000 hasta diciembre del 2020. La selección fue realizada por tres investigadores en dos etapas (cribado y aplicación de criterios de elegibilidad). Se registraron edad, sexo, tamaño muestral, calidad metodológica, variables, instrumento y resultados en 16 artículos que cumplieron los criterios de inclusión. Resultados: El 56,25\% de los estudios resaltan la insatisfacción corporal, así como el riesgo de padecer TCA en gimnastas de mayor nivel competitivo, resultando como factores causantes fundamentalmente la insatisfacción corporal y la presión del entorno sobre todo en el periodo de la adolescencia. El instrumento más utilizado fue Eating Attitude Test 26 (EAT-26) (75\%). Conclusiones: Los resultados obtenidos indican que existe gran riesgo en gimnastas de padecer TCA, destacando la aparición en categorías superiores, siendo una de las principales causas, la distorsión e insatisfacción con la IC.

Palabras clave. Imagen corporal, trastornos de conducta alimentaria, gimnasia, insatisfacción corporal, adolescentes.

Abstract. Introduction: In gymnastic sports, certain morphological measures such as weight, body mass index (BMI) and low-fat percentage are important for sporting success. Excessive preoccupation with these factors, or altered Body Image (BI), can lead to eating disorders (ED) in gymnasts. Objective: The purpose of this study was to analyse ED and CI in gymnastics athletes through a systematic review. Material and methods: Following the «PRISMA» checklist, five electronic databases (WOS, PubMed, Sports Discuss, Scopus and Google Scholar) were searched for cross-sectional studies in English, Spanish and Portuguese published from January 2000 to December 2020 Selection was performed by three investigators in two stages (screening and application of eligibility criteria). Age, sex, sample size, methodological quality, variables, instrument and results were recorded in 16 articles that met the inclusion criteria. Results: $56.25 \%$ of the studies highlight body dissatisfaction, as well as the risk of suffering from ED in gymnasts at a higher competitive level, with body dissatisfaction and environmental pressure being the main causal factors, especially in the adolescent period. The most commonly used instrument was the Eating Attitude Test 26 (EAT-26) (75\%). Conclusion: The results obtained indicate that gymnasts are at high risk of suffering from BAD, with a high incidence in the higher categories, one of the main causes being distortion and dissatisfaction with the CI.

Key words. Body image, eating disorders, gymnastics, body dissatisfaction, adolescents.

\section{Introducción}

La manera en que un individuo se percibe, imagina, siente y actúa respecto a su propio cuerpo es denominada como imagen corporal (IC). Esta puede ir cambiando a lo largo de la vida, siendo más acentuada en la adolescencia convirtiéndose esta etapa en una de las poblaciones de mayor riesgo de sufrir insatisfacción con

Fecha recepción: 07-09-21. Fecha de aceptación: 01-12-21

Alicia Salas

aliciasalasmorillas@gmail.com la IC. Igualmente, cada vez es más frecuente en poblaciones de menos edad (Trejo, Castro, Facio, Mollinedo, \& Valdez, 2010). En el estudio de Ariza-Vargas, SalasMorillas, López-Bedoya y Vernetta-Santana (2020), se observó que los gimnastas de acrobática a nivel base de competición presentaban mayor satisfacción corporal frente a un grupo control.

Por otro lado, los Trastornos de la Conducta Alimentaria (TCA) se caracterizan por anormalidades en el comportamiento de la ingesta de alimentos, teniendo como finalidad controlar el peso, cuya base se encuentra en una alteración psicológica. Estos TCA son 
importantes problemas de salud mental que afectan principalmente a mujeres jóvenes y se constituyen en trastornos severos casi siempre crónicos, con alta disfuncionalidad (Herpertz-Dahlmann, 2009).

Es fundamental analizar los factores de riesgo de estos TCA para su recuperación o prevención. Entre ellos se encuentran los relacionados con la insatisfacción corporal y la preocupación excesiva por el peso, así como la influencia que ejerce el entorno, padres, entrenadores y compañeros (Vaquero-Cristóbal, Alacid, Muyor \& López-Miñarro, 2013; Verdugo, Hernández Armas, Baños, Moncada-Jiménez \& Rentería, 2020).

Además del propio deporte y el nivel al que se practica, Picard (1999) apunta como factor de riesgo a la categoría a la que pertenece el deportista. En esta línea Wilmore y Costill, (1998) citado en Rodríguez, Fernández, Ruiz de Azua y Goñi (2005), indican que la prevalencia estimada de TCA en el ámbito deportivo aparece en un 50\% para deportistas de alta competición frente a $1 \%$ en la población en general. En los estudios de De Bruin, Oudejans \& Bakker (2007), Neves, Meireles, Carvalho, Almeida \& Ferreira (2016ab) y Verdugo, et al. (2020) se afirma así mismo, que las gimnastas tenían mas riego de sufrir TCA frente a población sedentaria.

La presión de cada deporte puede traer consigo riesgo de padecer TCA, en los deportes estéticos, entre los que se encuentran las disciplinas gimnásticas. La gran complejidad técnica y buenas cualidades físicas junto a una buena IC, son algunos de los determinantes en el éxito. En esta IC, ciertas medidas morfológicas como el peso, un IMC y un porcentaje de grasa bajo adquieren su importancia, convirtiéndose en procesos selectivos para dicho éxito (Fortes, Neves, Filgueiras, Almeida \& Ferreira, 2013; Taboada-Iglesias, Vernetta \& GutiérrezSánchez, 2017).

Una preocupación excesiva por el peso o alteración de la IC, puede llevar a estos gimnastas a desórdenes alimentarios produciendo efectos desfavorables como la disminución del rendimiento deportivo, y lo que es aún más importante, un impacto negativo para la salud. Otra de las mayores causas es el entorno (Kouloutbani, Komanthi,Theodosis Efstathiou \& Stergioulas Apostolos, 2012) y la internalización del cuerpo de un gimnasta (Fortes, Ferreira \& Almeida, 2014; Vieira, Amorin, Vieira, Amorin \& Rocha, 2009a) ya que los propios deportistas han pasado a ser referentes para adolescentes que practican deporte (Dosil \& Díaz, 2012). La influencia de los entrenadores juega un papel muy importante, ya que son los principales referentes dentro de su discipli- na gimnástica (Francisco, Alarcão \& Narciso, 2012). Son varios los estudios que analizan los TCA causados desde el punto de vista de la presión ejercida por el entrenador, se observó como los entrenadores presionaban a las gimnastas pesándolas muy a menudo, por lo que esto puede ser traducido para las gimnastas, en que un bajo peso corresponde a un buen trabajo dentro del entrenamiento (Ioannidou \& Venetsanou, 2019; Kosmidou, Giannitsopoulou \& Proios, 2018).

El riesgo para sufrirTCA es diferente entre gimnastas y no practicantes, debido a que los patrones que se consideran patológicos en un entorno más general, podrían ser más comunes e incluso funcionales en un contexto deportivo de élite (Bloodworth, McNamee \& Tan, 2017).

El término IC es un constructo muy empleado en el campo de la psicología y psiquiatría. Se considera crucial para explicar aspectos importantes de la personalidad, autoestima, autoconcepto, o para explicar la integración social de los adolescentes. Se enmarca en un continuo desde sentimientos positivos de apreciación y disfrute a sentimientos negativos de angustia u odio (MacLean \& Paxton, 2019). Ambos enfoques, tanto el negativo como el positivo son factores relevantes en el desarrollo de los TCA. De ahí, que el propósito de este estudio fue analizar los TCA ligados a la IC, en practicantes de deportes gimnásticos mediante una revisión sistemática.

Los resultados de esta revisión aportarán un mayor conocimiento de la posible relación de la IC en los TCA en los gimnastas, contribuyendo con esta información al actual corpus científico en este campo. Por lo que nuestro objetivo, se centra en analizar los estudios existentes ligados a la IC y los TCA en gimnastas.

\section{Material y métodos}

\section{Diseño del estudio}

Los estudios incluidos en la presente revisión sistemática de la literatura examinan los trastornos alimentarios y la IC en practicantes de deportes gimnásticos. Esta revisión sistemática se adhirió a la directriz Preferred Reporting Items for Systematic Reviews and MetaAnalysis PRISMA formada por cuatro fases: identificación, selección, elegibilidad e inclusión (Moher et al., 2015). Se utilizó la estrategia PICOS, la cual representa el acrónimo en inglés Population, Intervention, Comparison, Outcomes, Study- desing. Para esta investigación, «Population» hace referencia a deportistas gimnastas; «Intervention», a cualquier disciplina gimnástica; «Comparison» no aplica; «Outcomes», enfoca- 
do a la descripción de TCA e IC en deportes gimnásti$\cos \mathrm{y}$ «Study-desing» incluye aquellos estudios descriptivos. Estos elementos que componen dicha estrategia (PICOS), son fundamentales para la búsqueda bibliográfica (Roever, 2017). El protocolo fue registrado en la base de datos PROSPERO (Moher et al., 2015). (ref: CRD42021267953).

\section{Estrategias de búsqueda}

Se realizaron búsquedas en cinco bases de datos electrónicas, WOS, Pubmed, SPORTDiscus, Scopus y Google Scholar de artículos originales publicados desde el 1 enero del 2000 al 31 diciembre del 2020. Se utilizaron los siguientes términos de búsqueda y términos $\mathrm{MesH}$; «Eating disorders», «body image», «gymnastics», «sports gymnasts», «trastornos alimentarios», «imagen corporal», «gimnastas», «deportes gimnásticos» y combinando las palabras clave con los operadores booleanos AND y OR.

\section{Criterios de inclusión}

Los criterios de inclusión fueron: a) artículos originales en gimnastas (mujeres y varones); b) rango de edad de nueve a 21 años; c) que incluyesen las variables TCA e IC de forma conjunta; d) escrito en idioma inglés, español o portugués.

Se excluyeron todas las investigaciones que: a) incluáan trabajos anteriores al 2000; b) estudios de revisiones sistemáticas o meta-análisis; c) comunicaciones presentadas a congresos, capítulos de libros o tesis doctorales; d) estudios cuyo objetivo principal fuesen otros trastornos psiquiátricos diferentes a los TCA; e) estudios que incluían población fuera del rango de edad indicado o no gimnastas.

Este rango se acotó por dos condiciones fundamentales: la primera, porque los gimnastas se encuentran desarrollando plenamente su actividad deportiva en estas edades en diferentes niveles competitivos, y la segunda, porque los factores de riesgo de TCA tienen alta incidencia en esta etapa del desarrollo (Palacios de Espig \& Sánchez, 2016).

\section{Procedimiento de selección}

Siguiendo las normas PRISMA Moher et al. (2015) se extrajeron 103 artículos, una vez aplicados los criterios de selección, el trabajo se compuso de 16 artículos válidos. (Figura 1).

Todo el proceso de selección y evaluación de los artículos se hizo por tres revisores independientes (ASM; MVS; AGS), según las directrices de la Strengthening the
Reporting of Observational Studies in Epidemiology (STROBE), para verificar la rigurosidad y calidad técnica de las investigaciones. La lista contiene 22 ítems sobre lo que debe de incluirse en una descripción precisa de los estudios observacionales. Los estudios tienen una puntuación entre cero y 22 puntos. En caso de discrepancias entre los revisores en la evaluación de algún artículo, se volvía a evaluar hasta llegar al consenso. La puntuación metodológica se comparó con la puntuación máxima de STROBE clasificándose los artículos en tres grupos según su calidad metodológica: calidad alta (e» $70 \%$ de la puntuación total), calidad moderada, (50$69 \%$ puntuación total) y baja calidad $(<50 \%$ de la puntuación total) (Strong et al., 2005).

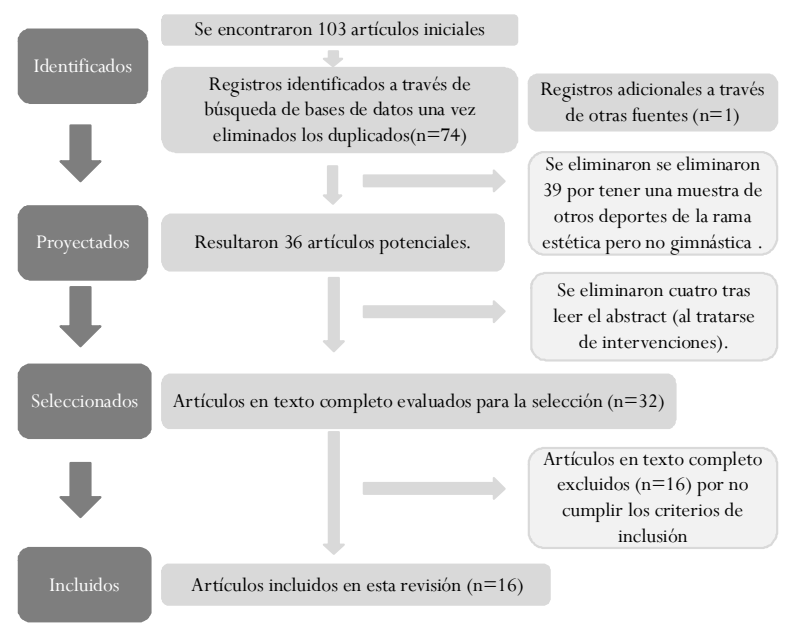

Figura 1. Diagrama de flujo de acuerdo a PRISMA.

\section{Extracción de datos}

Para el análisis de datos se realizó una tabla síntesis donde se registraron: autores y año, puntuación de la calidad metodológica, población de estudio (número de participantes, edad y sexo), variables estudiadas, test utilizados, así como los resultados más relevantes descritos de cada investigación.

\section{Resultados}

De acuerdo con el periodo evaluado se observa falta de investigación en el primer quinquenio (2000-2004), con la escasez en el segundo (2005-2009) con solo 2 estudios, experimentando un repunte importante en los dos últimos quinquenios, encontrándose 7 estudios en cada uno de ellos (Figura 2).

Las disciplinas más estudiadas han sido gimnasia artística $(50 \%)$ seguida de gimnasia rítmica $(18,7 \%)$, existiendo un solo estudio que hace comparativa con gimnasia aeróbica y rítmica (Ioannidou \& Venetsanou, 2019). El resto de los artículos no especifica la disciplina gimnástica. 


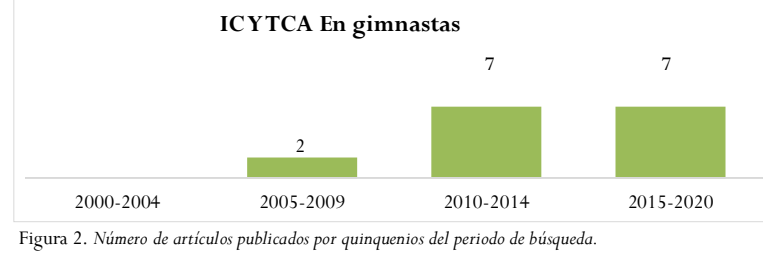

En la tabla 1 se presentan los resultados de forma sintética de los factores analizados de los 16 artículos seleccionados en esta revisión.

Se utilizaron diferentes test en cada uno de los estudios analizados, empleándose entrevistas en algunos de ellos, lo que dificultó la comparación de los resultados entre todos los estudios seleccionados (tabla 2). Los más utilizados fueron el EatingAttitude Test (75\%) y el Body Shape Questionnaire (50\%).

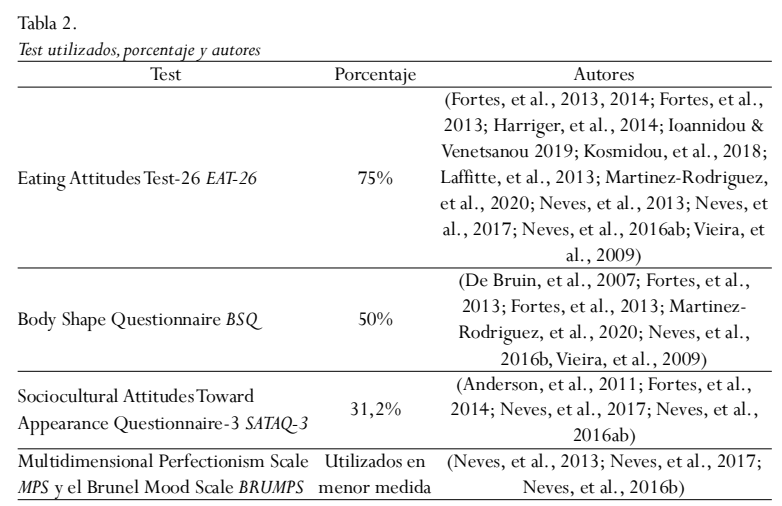

\section{Discusión}

Esta revisión sistemática trató de aportar información sobre los trastornos alimentarios y la insatisfacción corporal en los deportistas de las distintas disciplinas gimnásticas.

La discusión ha sido organizada de acuerdo a los siguientes criterios:

Idioma predominante y número de autores por artículos

El idioma predominante fue el inglés $(43,7 \%)$ y el portugués $(43,7 \%)$. El rango de autores es de dos a seis, predominando los estudios realizados con tres o cinco autores (37,5\% y 31,2\% respectivamente). $\mathrm{Al}$ analizar las autorías, se percibe una red de colaboración ya que los autores con mayor cantidad de artículos, los producen de forma conjunta. Esta tendencia ha sido evidenciada como próspera para los investigadores y para el campo científico (García, Martrucelli, Rossilho \& Denardin, 2010).

\section{Tamaño de la muestra edad y género}

El tamaño de la muestra global se enmarca entre 11 en el estudio de Laffitte, Zap, Leandro y Colleon (2013) hasta 828 en Anderson, Petrie y Neumann (2011). Con respecto a la edad, se trabajó con preadolescentes (9-12 años) y adolescentes (13-21 años). En todos los estudios se engloba diferentes rangos de edades, adolescentes y preadolescentes.

En relación al sexo, la mayoría de los artículos encontrados se han realizado en gimnastas femeninas $(81,2 \%)$ y el resto en ambos sexos, debido a la gran participación femenina en deportes gimnásticos en general. La mayor parte de los estudios se han centrado en la gimnasia rítmica, disciplina donde en la actualidad la Federación Internacional de Gimnasia solo recoge la modalidad femenina, aunque España es el único país del mundo que cuenta con un Campeonato Nacional de gimnasia rítmica masculina. No obstante, a pesar de que cada vez se va generalizando más la participación del género masculino en diferentes deportes, los estudios siguen siendo escasos (Taboada-Iglesias, Vernetta-Santana, Alonso-Fernández \& Gutiérrez-Sánchez, 2019).

\section{Metodología}

Con respecto a la calidad metodológica en función de la escala STROBE, la mayoría de los estudios que conforman esta revisión sistemática $(93,7 \%)$, mostraron una calidad alta, es decir la puntuación obtenida ha sido mayor al $70 \%$. Un solo estudio $(6,2 \%)$ presentó calidad moderada (Laffitte, et al, 2013) y ninguno calidad baja (tabla 1). El test más utilizado por el $75 \%$ de los estudios encontrados fue el EAT-26, Eating Attitude Test.

En cuanto a las variables analizadas, el 100\% de los estudios además de las variables principales a evaluar (TCA e IC), la mayoría $(68,7 \%)$ analizan también la composición corporal mediante medidas antropométricas (peso, talla e IMC), y seis de ellos $(37,5 \%)$ incluyen perímetro de cintura y pliegues cutáneos. En tres estudios (18,7\%) se analiza también presiones sociales (Anderson et al., 2011; Ioannidou \& Venetsanou, 2019; Kosmidou et al., 2018), tres artícu$\operatorname{los}(18,7 \%)$ perfeccionismo (Neves et al., 2016a; Neves, Filgueiras, Berbert de Carvalho, Schubring, BarkerRuchti, \& Caputo Ferreira, 2017; Neves, Filgueiras, Fortes \& Ferreira, 2013), dos artículos (12,5\%) estado de ánimo (Neves et al., 2013; Neves et al., 2017) y uno $(6,2 \%)$ la ansiedad (Fortes et al., 2013).

\section{Resultados encontrados}

Un total de cuatro estudios prospectivos (25\%) analizados han identificado la insatisfacción como la causan- 
Tabla 1.

\begin{tabular}{|c|c|c|c|c|}
\hline Autores & Calidad & Población de estudio & Variables estudiadas & Test \\
\hline $\begin{array}{l}\text { (De Bruin et } \\
\text { al., 2007) }\end{array}$ & $81,8 \%$ & $\begin{array}{c}70 \text { gimnastas de élite, } 51 \\
\text { gimnastas de no élite y } 85 \text { no } \\
\text { practicantes, } \\
(15 \text { a } 17 \text { años }) \\
\end{array}$ & $\begin{array}{c}\text { Imagen corporal (IC) } \\
\text { Medidas de comportamiento de la } \\
\text { dieta. }\end{array}$ & $\begin{array}{c}\text { BSQ } \\
\text { BULIT-R }\end{array}$ \\
\hline $\begin{array}{l}\text { (Vieira et al., } \\
2009 \text { ) }\end{array}$ & $81,8 \%$ & $\begin{array}{l}48 \text { gimnastas de rítmica } \\
\text { (categorías; infantil, juvenil y } \\
\text { senior) y } 48 \text { estudiantes (grupo } \\
\text { control) } \\
\text { (10-18 años) }\end{array}$ & $\begin{array}{c}\text { IC } \\
\text { TCA } \\
\text { Variables antropométricas }\end{array}$ & $\begin{array}{c}\text { BSQ } \\
\text { EAT-26 }\end{array}$ \\
\hline $\begin{array}{l}\text { (Anderson et } \\
\text { al., 2011) }\end{array}$ & $72,7 \%$ & $\begin{array}{l}\text { 414 NCAA (liga universitaria } \\
\text { en EEUU), División I; } 280 \\
\text { gimnastas universitarias y } 134 \\
\text { nadadores y buzos. } \\
\text { (M edad }=19,14 \text { años) }\end{array}$ & $\begin{array}{c}\text { Satisfacción corporal. } \\
\text { Internalización del ideal de delgadez. } \\
\text { Presiones de peso deportivo. } \\
\text { Presiones socioculturales generales. } \\
\text { Restricción de la dieta. } \\
\text { Afecto negativo. } \\
\text { Comportamientos modelados. } \\
\text { Síntomas de bulimia } \\
\text { Deseabilidad social. } \\
\text { Variables demográficas. } \\
\end{array}$ & $\begin{array}{l}\text { BPSS-R } \\
\text { SATAQ-3 } \\
\text { WPS } \\
\text { PSPS } \\
\text { DIS } \\
\text { PANAS-X } \\
\text { BULIT-R } \\
\text { MCSD }\end{array}$ \\
\hline $\begin{array}{c}\text { (Fortes et al., } \\
2013)\end{array}$ & $77,2 \%$ & $\begin{array}{l}62 \text { gimnastas de artística } \\
\text { femeninas. } \\
\text { (12-17 años) }\end{array}$ & $\begin{array}{c}\text { IC } \\
\text { TCA } \\
\text { Ansiedad } \\
\text { Variables antropométricas }\end{array}$ & $\begin{array}{c}\text { BSQ } \\
\text { EAT-26 } \\
\text { STAI }\end{array}$ \\
\hline $\begin{array}{c}\text { (Fortes et al., } \\
2013 \text { ) }\end{array}$ & $90,9 \%$ & $\begin{array}{c}47 \text { atletas (gimnastas de } \\
\text { artística y nadadoras de } \\
\text { sincronizada) entre } 12-16 \text { años }\end{array}$ & $\begin{array}{l}\text { Insatisfacción corporal } \\
\text { Nivel de compromiso psicológico para } \\
\text { hacer ejercicio }\end{array}$ & $\begin{array}{c}\text { BSQ } \\
\text { EAT-26 } \\
\text { CES }\end{array}$ \\
\hline
\end{tabular}

\begin{tabular}{|c|c|c|c|}
\hline $\begin{array}{l}\text { (Laffitte et al., } \\
\text { 2013) }\end{array}$ & $59,09 \%$ & $\begin{array}{c}11 \text { gimnastas femeninas de } \\
\text { rítmica } \\
\text { Selección Paranaense } \\
(12-14 \text { años })\end{array}$ & $\begin{array}{c}\text { IC } \\
\text { TCA } \\
\text { Gravedad síntomas bulímicos } \\
\text { Variables antropométricas }\end{array}$ \\
\hline
\end{tabular}

Las gimnastas de élite hicieron dieta con más frecuencia que las no practicantes, pero fueron menos negativas en relación a su IC. de no élite hicieron dists tonto como las no practicantes y tenía una IC más positiva. Respecto a TCA no hubo diferencias significativas entre ambos grupos.
Hubo una correlación significativa entre los TCA y la distorsión de la IC de las gimnastas en las categorías infantil (10 a 12 años) y Hubo una correlación significativa entre los TCA y la distorsión de la IC de las gimnastas en las categorías infantil (10 a 12 años) y
juvenil (13 a 14 años). juvenil (13 a 14 años).

actitudes que sugerian un comportamiento de desorden alimenticio. Todas las gimnastas mayores de 15 años presentaron
and and distorsió La percepción conductual y cognitiva de todos los adolescentes en relación con las exigencias estéticas de los entornos en los que se encuentran, parece ser un factor primordial para la presencia de conductas que pueden llevar a TCA y a distorsión de la IC.

Específicamente, las presiones sociales generales con respecto al peso y al cuerpo estaban relacionadas con la internalización de esos ideales y, subjetivamente, con el aumento de la insatisfacción corporal.

Las presiones del entorno deportivo en relación con el peso y la apariencia estaban asociadas con una mayor insatisfacción corporal y una alimentación más restrictiva.

La insatisfacción corporal estaba relacionada con más sentimientos de tristeza, ira y miedo entre los atletas.

El afecto negativo, la insatisfacción corporal y la restricción dietética se relacionaron directamente con los síntomas bulímicos, lo que explica el $55-58 \%$ de su variación. Ter aún más generalizadas y negativas para las mujeres atletas.

Influencia estadísticamente significativa sólo del STAI- $S$ en elTCA ( $F(1,61)=4,56 ; \mathrm{p}=0,047)$.

Mayor puntuación en el STAI-T en las gimnastas con TCA cuando se compararon con las que no tenían riesgo de padecer trastornos alimentarios $(\mathrm{F}(1,61)=8,59 ; \mathrm{P}=0,009)$.

La ansiedad estado influý en los comportamientos de riesgo de padecerTCA de las jóvenes gimnastas.

También hay que señalar que las gimnastas con TCA demostraron mayor ansiedad rasgo.

Los resultados del BSQ mostraron no insatisfacción corporal en las gimnastas en la muestra en general.

Asociación significativa entre insatisfacción corporal y comportamiento alimentario y entre LPCE (nivel de compromiso psicológico con el ejercicio) y riesgo comportamiento para trastornos alimenticios en atletas de deportes estéticos

Las gimnastas no alcanzaron la recomendación del porcentaje mínimo de grasa de este grupo de edad, siendo el $81,1 \%$ las que alcanzaron el valor medio de la modalidad.

El consumo de más de la mitad de la muestra alcanzó el mínimo energético, proteínico y lipídico recomendado, y menos de la mitad alcanzó el mínimo de carbohidratos, calcio y hierro.

BSQ El $27,1 \%$ de las gimnastas presentaban riesgo de anorexia, y un $63,5 \%$ riesgo de bulimia

BITE Baja correlación de ingesta alimentaria y porcentaje de grasa corporal. Correlación; con mayor consumo energético más satisfechas con su IC, porcentaje de grasa corporal por encima de la media y mayor riesgo de padecer bulimia.

Las gimnastas presentan un porcentaje de grasa corporal inferior al recomendado para su edad y, dentro de lo normal para los deportes, una ingesta alimentaria inadecuada, que puede provocar daños en la salud, mayor tendencia a la bulimia que a la anorexia insatisfacción con el cuerpo.

\begin{tabular}{|c|c|c|c|c|c|}
\hline $\begin{array}{l}\text { (Neves et al., } \\
2013 \text { ) }\end{array}$ & $81,8 \%$ & $\begin{array}{l}50 \text { gimnastas de artística de } \\
\text { alto rendimiento } \\
\text { (10-15 años) }\end{array}$ & $\begin{array}{l}\text { Insatisfacción corporal } \\
\text { TCA } \\
\text { Perfeccionismo. } \\
\text { Estado de ánimo. } \\
\text { Variables antropométricas. }\end{array}$ & $\begin{array}{l}\text { BSQ } \\
\text { EAT-26 } \\
\text { MPS } \\
\text { BRUMS }\end{array}$ & $\begin{array}{l}\text { No existe ninguna asociación significativa de perfeccionismo con TCA ( }>>0,05) \text {, sin embargo, el segundo modelo de regresión } \\
\text { múltiple indicó una relación estadísticamente significativa de la subescala de fatiga de BRUMS en las puntuaciones del EAT-26 ( } \mathrm{F}(\text {, } \\
49 \text { ) }=5,09 \text {; } \mathrm{p}=0,048 \text { ). Se concluye que, aunque los comportamientos perfeccionistas no inducen a los gimnastas a la TCA, la } \\
\text { sensación de fatiga puede predisponer a estos atletas a la TCA. }\end{array}$ \\
\hline $\begin{array}{l}\text { (Fortes et al., } \\
\text { 2014) }\end{array}$ & $77,2 \%$ & $\begin{array}{l}62 \text { gimnastas femeninas de } \\
\text { artística. } \\
(12-17 \text { años. })\end{array}$ & $\begin{array}{c}\text { TCA } \\
\text { Internalización del ideal de delgadez }\end{array}$ & $\begin{array}{c}\text { EAT-26 } \\
\text { SATAQ-3 }\end{array}$ & $\begin{array}{l}\text { No se observó ninguna influencia de las subescalas del SATAQ-3 en la restricción de alimentos }(\mathrm{p}>0,05) \text { y el autocontrol oral } \\
(\mathrm{p}>0,05) \text {, sin embargo, indicaron la influencia de la internalización de la delgadez en los síntomas bulímicos }(\mathrm{p}<0,05) \text {. } \\
\text { Se puede concluir que la internalización ideal del cuerpo fue un importante predictor de los síntomas de la bulimia. }\end{array}$ \\
\hline $\begin{array}{l}\text { (Harriger et al., } \\
\text { 2014) }\end{array}$ & $81,8 \%$ & $\begin{array}{l}100 \text { gimnastas femeninas } \\
(10-15 \text { años })\end{array}$ & $\begin{array}{l}\text { TCA } \\
\text { IC } \\
\text { Demographics and gymnastics } \\
\text { participation. }\end{array}$ & $\begin{array}{l}\text { EAT-26 } \\
\text { CHEAT } \\
\text { Rosenberg Self } \\
\text { Esteem Scale } \\
\text { OBC-Youth } \\
\text { Pubertal } \\
\text { Development } \\
\text { Scale }\end{array}$ & $\begin{array}{l}\text { El desarrollo de la pubertad se asocia con mayores niveles de vigilancia corporal, vergüenza corporal y trastornos alimentarios. } \\
\text { * El mayor tiempo dedicado al entrenamiento se asociaba con menores niveles de vergüenza corporal y trastornos alimentarios. } \\
\text { * La mayor autoestima se asoció con menores niveles de trastornos alimentarios, menos vigilancia corporal y menos vergüenza } \\
\text { corporal. }\end{array}$ \\
\hline
\end{tabular}

Los gimnastas de élite presentaron un menor IMC y \%G.
En las subescalas del SATAQ-3, los gimnastas de élite presentaron un valor menor de "presión" y un valor mayor de En las subescalas del SATAQ-3, los gimnastas de élite presentaron un valor menor de "presión" y un valor mayor de
"internalización atlética" que los otros grupos. "internalización atlética" que los otros grupos.
Del análisis de regresión para los gimnastas base, las subescalas "internalización general" y "presión" fueron juntas, responsables

\begin{tabular}{|c|c|c|c|}
\hline $\begin{array}{c}\text { (Neves et al., } \\
2006 a)\end{array}$ & $86,3 \%$ & $\begin{array}{c}413 \text { adolescentes; } \\
\text { Gimnastas de artística elite, } \\
\text { gimnastas base, grupo control. } \\
\text { (10-18 años) }\end{array}$ & $\begin{array}{c}\text { TCA } \\
\text { Influencia del deporte y el ejercicio } \\
\text { sobre la IC. } \\
\text { Variables antropométricas. }\end{array}$ \\
\hline
\end{tabular}

\begin{tabular}{|c|c|c|c|c|}
\hline & & 413 adolescentes: 40 & Insatisfacción corporal & \\
\hline $\begin{array}{l}\text { (Neves et al., } \\
2006 \mathrm{~b})\end{array}$ & $77,2 \%$ & $\begin{array}{l}\text { deportistas de alto rendimiento } \\
\text { ( } 37 \text { chicas); } 245 \text { practicantes } \\
\text { de gimnasia artística base ( } 207 \\
\text { chicas) y } 128 \text { adolescentes no } \\
\text { deportistas ( } 60 \text { chicas). } \\
10-18 \text { años }\end{array}$ & $\begin{array}{c}\text { TCA } \\
\text { Influencia del deporte y el ejercicio } \\
\text { sobre la IC. } \\
\text { Perfeccionismo. } \\
\text { Estado de ánimo. } \\
\text { Variables antropométricas. }\end{array}$ & $\begin{array}{l}\text { BSQ } \\
\text { EAT-26 } \\
\text { SATAQ-3 } \\
\text { MPS } \\
\text { BRUMS }\end{array}$ \\
\hline $\begin{array}{c}\text { (Neves et al., } \\
\text { 2017) }\end{array}$ & $86,3 \%$ & $\begin{array}{l}20 \text { gimnastas artística } \\
\text { brasileñas } \\
\text { Mujeres } \\
\text { (10-16 años })\end{array}$ & $\begin{array}{c}\text { IC } \\
\text { TCA } \\
\text { Influencia del deporte y el ejercicio } \\
\text { sobre la IC. } \\
\text { Perfeccionismo. } \\
\text { Estado de ánimo. } \\
\text { Variables antropométricas. }\end{array}$ & $\begin{array}{c}\text { BSQ } \\
\text { EAT-26 } \\
\text { SATAQ-3 } \\
\text { MPS } \\
\text { BRUMS }\end{array}$ \\
\hline $\begin{array}{l}\text { (Kosmidou et } \\
\text { al., 2018) }\end{array}$ & $81,8 \%$ & $\begin{array}{c}3 \text { gimnastas de rítmicas } \\
\text { griegas. } \\
34 \text { ex gimnastag y } 49 \text { gimnastas } \\
\text { actuales. } \\
\text { (Ex gimnastas,20-30 años; } \\
\text { gimnastas, 10-14 años) }\end{array}$ & $\begin{array}{c}\text { IC } \\
\text { TCA } \\
\text { Presión por un cuerpo delgado por } \\
\text { parte de los entrenadores, padres y } \\
\text { amigos. } \\
\text { Variables antropométricas }\end{array}$ & $\begin{array}{c}\text { BES } \\
\text { EAT-26 }\end{array}$ \\
\hline $\begin{array}{l}\text { (Ioannidou \& } \\
\text { Venetsanou, } \\
\text { 2019) }\end{array}$ & $86,3 \%$ & $\begin{array}{l}90 \text { chicas gimnastas; } 41 \text { atletas } \\
\text { de gimnasia rítmica femenina } \\
\text { (RG) y } 49 \text { de gimnasia aeróbica } \\
\text { (AG) a nivel competitivo } \\
\text { ( } \mathrm{M} \text { edad }=13,9 \text { años) }\end{array}$ & $\begin{array}{c}\text { TCA } \\
\text { Presión por un cuerpo delgado por } \\
\text { parte de entrenadores, padres y } \\
\text { amigos. } \\
\text { Variables antropométricas }\end{array}$ & $\begin{array}{c}\text { EAT-26 } \\
\text { SPAS }\end{array}$ \\
\hline $\begin{array}{l}\text { (Martínez- } \\
\text { Rodríguez et } \\
\text { al., 2020) }\end{array}$ & $81,8 \%$ & $\begin{array}{c}33 \text { gimnastas femeninas de } \\
\text { rítmica } \\
(11-18 \text { años })\end{array}$ & $\begin{array}{c}\text { IC } \\
\text { Comportamiento Alimentario } \\
\text { Composición corporal } \\
\text { Estado nutricional }\end{array}$ & $\begin{array}{c}\text { BSQ } \\
\text { EAT-26 } \\
\text { Medidas } \\
\text { antropométricas }\end{array}$ \\
\hline
\end{tabular}

AT-26 del 12,4\% de la varianza de la conducta de riesgo para el desorden alimenticio.

SATAQ-3 Para los gimnastas de élite, las subescalas "internalización atlética" e "información" explicaban el 17,6\% de estos

A partir de los resultados de la correlación de las subescalas del SATAQ-3 con el EAT-26 para el grupo de control, no se identificaron asociaciones significativas

Los aspectos mediáticos que influyen en las conductas de riesgo de los trastornos alimentarios son diferentes en los gimnastas base

$\mathrm{y}$ de élite.
Los no deportistas presentaron mayor IMC que los gimnastas base $(\mathrm{p}=0,0001)$ y de alto rendimiento $(\mathrm{p}=0,0001)$.

Los no deportistas presentaron mayor IMC que los gimnastas base $(\mathrm{p}=0,0001)$ y de alto rendimiento $(\mathrm{p}=0,0001)$.
$\mathrm{El} \%$ de grasa corporal de los gimnastas de alto rendimiento fue significativamente menor que los no deportistas $(\mathrm{p}=0,0001)$ y los stas base $(\mathrm{p}=0,0001)$

-3 Perfeccionismo los gimnastas presentaron menor valor que los gimnastas base $(\mathrm{p}=0,0001)$.

Los gimnastas de base y no atletas presentaron menor desorden de ánimo que los de alto rendimiento ( $\mathrm{p}=0,007$ y $\mathrm{p}=0,041$

El riesgo de padecer TCA es el factor que más se relaciona con la insatisfacción corporal en todos las gimnastas.

Durante la temporada de competición, la insatisfacción corporal era mayor.

En la temporada de pre-competición, eran mayores; los desordenes alimentarios, el perfeccionismo y los valores de vigot.

Se encontraron que los desórdenes alimentarios son el predictor más fuerte de la insatisfacción corporal durante todas la estaciones, y el estado de ánimo contribuyó a la insatisfacción corporal en la temporada de competición.

La estima corporal se predijo significativamente por la presión de ser delgado por los padres, el IMC y la edad de entrenamiento. Las ex gimnastas tenían una estima corporal y actitudes alimentarias más positivas que las gimnastas actuales, y estas sentín más presión para estar delgados por parte de sus padres, que las ex gimnastas.

No hubo diferencias significativas entre gimnasta de rítmica y aerobic con respecto a SPA y TCA, sin embargo, las atletas de aerobic tenían una presión parental más alta para el cuerpo delgado que las de rítmica.

Cuando los datos de ambas disciplinas se fusionaron, se reveló un alto porcentaje (40\%) de TCA. Las gimnastas con TCA tuvieron significativamente más ansiedad mediante el SPA y presión percibida para el cuerpo delgado que aquellas con actitudes y comportamientos alimentarios normales.

Un elevado porcentaje de gimnastas presentan una preocupación por el peso en relación con la ingesta de alimentos. El 37,85\%

EAT-26 presentaban insatisfacción corporal. No obstante, se detectaron solo dos casos de riesgo de TCA y preocupación por la IC. Medidas Existió relación entre el riesgo de TCA con
antropométricas como el cansancio y dormir menos horas.

Al realizar una prueba ANOVA de dos factores, la insatisfacción con la IC, presento diferencias estadísticamente significativas por $\operatorname{sexo}(\mathrm{F} 1,55=17.41, \mathrm{p} d) 0.001)$ y grupo $(\mathrm{F} 2,55=3.66, \mathrm{p}=0.033)$, mostrando un mayor grado de insatisfacción en los hombres $(M=-0.54)$ respecto a las mujeres $(M=0.43)$.

\begin{tabular}{|c|c|c|}
\hline $\begin{array}{l}\text { (Verdugo et al., } \\
2020)\end{array}$ & $\begin{array}{l}56 \text { participantes (gimnastas } \\
\text { élite, gimnastas regulares y } \\
\text { grupo control) }\end{array}$ & $\begin{array}{c}\text { IC } \\
\text { TCA } \\
\text { Variables antropométricas. }\end{array}$ \\
\hline
\end{tabular}

Eliesgo de anorexia mostró diferencias significativas por $\operatorname{sexo}(\mathrm{F} 1,55=10.69, \mathrm{p}=0.002)$ y grupo $(\mathrm{F} 2,55=7.97, \mathrm{p}>0.001)$ Los gimnastas mayor grado de insatisfacción corporal (desean una silueta más gruesa o musculosa para el caso de los varones, anorexia en comparación con el grupo control.

EDI-2: eatingdisorderinventory; EAT-26: eatingattitude Test; BES-C: bodyesteemscaleforchildren; CES: Commitment to exercisescale; BSQ: bodyshapequestionnaire; Test; SATAQ-3: attitudestowardsappearance Questionnaire-3; MPS: Multidimensional perfectionismscale; BRUMS: brunelmoodscale; BULIT:R: Bulimia Test-Revised; BPSS-R:bodypartssatisfactionscale; STAI: State-traitanxiety inventory; SPAS: Social physiqueanxietyscale; BITE:bulimicinvestigatory Test Edimburgh; BES: Bodyesteem; EFRATA: Escala de Factores de Riesgo de Trastornos Alimentarios; WPS: Escala de Presiones de Peso de 20 elementos; PSPS: Escala de Presiones Socioculturales Percibidas de 35 elementos; DIS: Escala de Intención Dietética de 9 elementos; PANAS-X: Formulario Ampliado de la Lista de Efectos Positivos y Negativos; MSCD: Escala de Deseabilidad Social de Marlowe-Crowne de 12 elementos; CHEAT: Children'sEatingAttitudes Test; OBC-YOUTH: objectifiedbodyconsciousness - youthscale. 
te de riesgo para padecer TCA. Vieira, Vieira, Amorin, Amorin y Rocha (2009b) observan una correlación entre los TCA y distorsión de la IC en categorías Infantil (10-12años) y Juvenil (13-14 años). Anderson etal. (2011) observaron que la insatisfacción corporal y la restricción dietética se relacionaron directamente con los síntomas bulímicos, siendo varios estudios posteriores los que afirman esta relación (Fortes et al., 2013; Laffitte et al., 2013). Por otro lado, 12,5\% de los estudios atribuyen la causa del riesgo a otros factores como entorno, presión, ansiedad, etc., no observando la relación entre IC con TCA como en los estudios anteriores (Fortes et al., 2013; Vieira et al., 2009b).

De forma pormenorizada, referente a la IC, en la etapa de la pubertad se observa una preocupación por el peso (Harriger, Witherington \& Bryan, 2014), lo que implica la aparición de la insatisfacción corporal. Se puede diferenciar entre percepción de la IC y satisfacción con esta.Algunos de los estudios concluyen que los gimnastas poseen mejor percepción de la IC con respecto a no practicantes (De Bruin et al., 2007; Fortes et al., 2013), y entre grupos de gimnastas destacan los de élite con mejor percepción de esta (De Bruin et al., 2007). Sin embargo, no concuerda con los resultados obtenidos en los estudios de Neves et al. (2017) yVerdugo et al. (2020), donde los gimnastas distorsionaban su IC, siendo en las gimnastas de rítmica donde más ocurría (Vieira et al., 2009a).

En cuanto a la insatisfacción, los estudios afirman que generalmente se dan en gimnastas mayores de 15 años (Vieira et al., 2009b), en temporada de competición (Neves et al., 2017) y resultaba mayor en el género masculino difiriendo del sexo femenino por querer tener un físico más musculoso y masculino (Verdugo et al., 2020). Las causas analizadas que conllevan a esta insatisfacción son las siguientes: presiones sociales en el entorno deportivo que se asocian con la interiorización de los ideales de belleza y corporales establecidos para gimnastas, así como sentimientos de ira, tristeza y miedo precedidos en mayor medida de atracones alimentarios y un nuevo compromiso de restricción de ingesta de alimentos. Todo esto incide sobre la insatisfacción corporal en primer lugar, que afecta posteriormente a comportamientos de restricción de la ingesta o comportamientos bulímicos (Anderson et al., 2018).

En cuanto a la variable de TCA, se observa un gran riesgo dentro de las disciplinas gimnásticas más latente en gimnasia rítmica y gimnasia aeróbica (Ioannidou \& Venetsanou, 2019; Laffitte et al., 2013; Verdugo et al., 2020), siendo mayor el riesgo en gimnastas de élite fren- te a gimnastas de base (Neves et al., 2016a). Dentro de los diferentes trastornos alimentarios existentes, el más común dentro de los gimnastas es la bulimia (Laffitte et al., 2013). El riesgo para sufrir TCA es diferente en función del momento de la temporada, siendo mayor en periodos pre-competitivos (Neves et al., 2017). Las causas que fomentan el riesgo a sufrir TCA, son entre otras; mayor IMC, distorsión de la IC, así como insatisfacción corporal (Harriger et al., 2014; Neves et al., 2016b).

Con respecto al IMC y porcentaje de grasa es menor en gimnastas de élite (Neves et al., 2016a; Neves et al., 2016b), destacando a las gimnastas de rítmica que poseen menor porcentaje graso recomendado para su edad (Laffitte et al., 2013). En el estudio de Laffite et al. (2013), relacionan un porcentaje de grasa bajo con una mayor satisfacción corporal, así como, un porcentaje graso por encima de la media una mayor tendencia al desarrollo de bulimia.

Otras variables analizadas que dan respuesta a la aparición o al riesgo de sufrir TCA, son la ansiedad y el perfeccionismo. Con respecto a la ansiedad, Fortes et al. (2013) afirman que la ansiedad estado es causa de riesgo y observa que los gimnastas que padecen TCA poseen una ansiedad rasgo mayor. Igualmente, parece que las gimnastas con TCA presentan más ansiedad físico social y presión percibida por tener un cuerpo delgado que aquellas con actitudes y comportamientos alimentarios normales (Strong et al., 2005). En cuanto al perfeccionismo variable poco estudiada parece no afectar al riesgo de padecer TCA (Neves et al., 2013).

\section{Conclusiones}

Este estudio busco describir las evidencias científicas desarrolladas en las dos últimas décadas (2000-2020) respecto a la IC y su relación con TCA en gimnastas, con el objetivo de generar información relevante para los profesionales (entrenadores, padres y compañeros) que intervienen directamente en el desarrollo de estos gimnastas. En la última década, desde el 2011 ha habido un repunte en los estudios en esta población.

A raíz de los resultados analizados de la presente revisión, se puede concluir que existe gran riesgo en gimnastas de rítmica y artística en padecer TCA, destacando la aparición en categorías superiores, siendo una de las principales causas, la distorsión e insatisfacción con la IC. En la etapa de la pubertad se observa mayor preocupación por el peso, siendo más acentuado en el género femenino, que finalmente se traduce en riesgo 
de sufrir TCA.

La importancia de estos conocimientos sirve para ser más conscientes de llevar un control de evaluación continuo sobre el estado nutricional y la ingesta dietética de estos deportistas, para detectar posibles riesgos de TCA y poder intervenir en caso necesario.

Con esto se contribuye a la salud y nutrición de los gimnastas, garantizando así, un buen desempeño en el ámbito deportivo.

\section{Limitaciones y futuras líneas de investiga- ción}

Nuestra principal limitación ha sido la falta de estudios encontrados que analicen en profundidad las disciplinas gimnásticas, es decir, que comparen gimnastas de diferentes disciplinas en función de la edad, género y nivel competitivo. Igualmente, la variedad de instrumentos de evaluación utilizados, lo que dificulta comparar en profundidad los resultados encontrados. No obstante, destacar la aparición de un mayor número de estudios en estos últimos diez años lo que ha facilitado este trabajo.

Como futuras líneas, sería necesario realizar estudios de carácter transversal analizando las variables deseadas en diferentes disciplinas gimnásticas, homogeneizando los instrumentos de medida. Igualmente, indagar sobre estudios de carácter longitudinal, para poder conocer la evolución de ambas variables y proponer posibles soluciones a esta problemática, así como, realizar estudios meta-analíticos para valorar el tamaño del efecto de los estudios y que las diferencias estadísticas no se deban a la variedad de instrumentos de evaluación utilizados, lo que dificulta comparar en profundidad los resultados encontrados. En definitiva, la revisión sistemática realizada pretende abrir camino a posteriores estudios más específicos en el ámbito de las disciplinas gimnásticas, abarcando las menos estudiadas y su influencia en el estado de salud de la población de gimnastas.

\section{Conflicto de intereses}

Los autores expresan que no existen conflictos de interés al redactar el manuscrito.

\section{Contribución específica}

Todos los autores han participado en el diseño del método, análisis de la información, obtención de resul- tados y tablas, en la generación de conclusiones y discusión, y trasmiten su conformidad a los criterios de autoría de los firmantes y su filiación profesional. No obstante, el peso de ASM ha sido fundamentalmente en la búsqueda bibliográfica en las diferentes bases, selección y evaluación de artículos, participación en la redacción, elaboración de figuras y tablas y preparación del formato del artículo y envío del mismo; AGS en la revisión y decisión de los artículos junto con MVS para verificar su inclusión y en la redacción y correcciones del mismo; MVS en el concepto intelectual del artículo (idea original, estructura del artículo y su coordinación), redacción, participación como revisora en la inclusión de los artículos y corrección crítica de la versión final para su aprobación antes del envío.

\section{Financiación}

Los autores expresan que no ha existido financiación para realizar este estudio.

\section{Referencias}

Anderson, C. M., Petrie, T.A., \& Neumann, C. S. (2011). Psychosocial correlates of bulimic symptoms among NCAA Division-I Female Collegiate Gymnasts and Swimmers/Divers. Journal of Sport and Exercise Psychology, 33(4), 483-505. https://doi.org/ 10.1123/jsep.33.4.483.

Ariza-Vargas, L., Salas-Morillas, A., López-Bedoya, J., \&Vernetta-Santana, M. (2020). Percepción de la imagen corporal en adolescentes practicantes y no practicantes de gimnasia acrobática (Perception of body image in adolescent participants and non-participants in acrobatic gymnastics). Retos, 39, 71-77. https: / / doi.org/10.47197/retos.v0i39.78282

Bloodworth, A., McNamee, M., \& Tan, J. (2017). Autonomy, eating disorders and elite gymnastics: ethical and conceptual issues. Sport, Education and Society, 22(8), 878-889. https://doi.org/10.1080/ 13573322.2015 .1107829$.

De Bruin, A. K., Oudejans, R. R., \& Bakker, F. C. (2007). Dieting and body image in aesthetic sports: a comparison of dutch female gymnasts and nonaesthetic sport participants. Psychology of Sport and Exercise 8(4), 507-520. https://doi.org/10.1016/ j.psychsport.2006.10.002.

Dosil, J., \& Díaz, I. (2012). Trastornos de Alimentación En Deportistas de Alto Rendimiento. Consejo Superior de Deportes. Madrid. 
Fortes, L., Almeida, S., \& Ferreira, M. E. (2013). Influência da ansiedade nos comportamentos de risco para os transtornos alimentares em ginastas. Revista Brasileira de Atividade Física \& Saúde, 18(5), 546553 https: / / doi.org/10.12820/rbafs.v. 18n5p546.

Fortes, L., Almeida, S., \& Ferreira, M. E. (2014). A internalização do ideal de magreza afeta os comportamentos alimentares inadequados em atletas do sexo feminino da Ginástica Artística?. Revista Da Educacao Fisica, 25(2), 181-191. https: / /doi.org/ 10.4025/reveducfis.v25i2.21866.

Fortes, L. D. S., Neves, C. M., Filgueiras, J. F., Almeida, S. S., \& Ferreira, M. E. C. (2013). Body dissatisfaction, psychological commitment to exercise and eating behavior in young athletes from aesthetic sports. Revista Brasileira de Cineantropometría \& Desempenho Humano, 15(6), 695-704. https:// doi.org/10.5007/1980-0037.2013v15n6p695.

Francisco, R., Alarcão, M., \& Narciso, I. (2012). Aesthetic sports as high-risk contexts for eating disorders young elite dancers and gymnasts perspectives. The Spanish Journal of Psychology, 15(1), 265-274. https:/ /doi.org/10.5209/rev_sjop.2012.v15.n1.37333.

Garcia, C. C., Martrucelli, C. R. N., Rossilho, M. D. M. F., \& Denardin, O.V.P. (2010). Autoria em artigos cientificos: os novos desafios. Brazilian Journal of Cardiovascular Surgery, 25(4), 559-567. https:// doi.org/10.1590/S0102-76382010000400021.

Harriger, J. A., Witherington, D. C., \& Bryan, A. D. (2014). Eating pathology in female gymnasts: potential risk and protective factors. Body Image, 11(4), 501-508. https://doi.org/10.1016/ j.bodyim.2014.07.007.

Herpertz-Dahlmann, B. (2009). Adolescent eating disorders: definitions, symptomatology, epidemiology and comorbidity. Child and Adolescent Psychiatric Clinics of North America 18(1), 31-47. https://doi.org/10.1016/j.chc.2008.07.005.

Ioannidou, C., \& Venetsanou, F. (2019). Social physique anxiety, disturbed eating attitudes and behaviors, and perceived pressure for thin body in competitive Rhythmic and Aerobic Gymnasts. Science of Gymnastics Journal, 11(3), 331-342.

Kosmidou, E., Giannitsopoulou, E., \& Proios, M. (2018). Are body esteem, eating attitudes, pressure to be thin, body mass index and training age related in rhythmic gymnastics athletes?. Science of Gymnastics Journal, 10(2), 189-201.

Kouloutbani, Komanthi, Theodosis Efstathiou, \& Stergioulas Apostolos. (2012). Eating disorders in the world of sport: the experiences of Rhythmic Gymnasts. Journal Biology of Exercise, 8(2), 19-31. https://doi.org/10.4127/jbe.2011.0057.

Laffitte, A. M., Zap, M., Leandro, P. P., \& Colleon, P. G. K. (2013). Relação entre composição corporal, consumo dietético e tendência a transtornos alimentares em atletas da seleção paranaense de Ginástica Rítmica. RBNE-Revista Brasileira de Nutrição Esportiva, 7(38), 99-107.

Martínez-Rodríguez, A., Reche-García, C., Martínez Fernández, M. C., \& Martínez Sanz, J. M. (2020). Valoración del estado dietético-nutricional, la composición corporal, el comportamiento alimentario y la percepción de la imagen en deportistas de gimnasia rítmica. Nutrición Hospitalaria, 37(6), 1217-1225. https://doi.org/10.20960/nh.03141.

McLean, S. A., \& Paxton, S. J. (2019). Body image in the context of eating disorders. Psychiatric Clinics, 42(1), 145-156. https://doi.org/10.1016/ j.psc.2018.10.006.

Moher, D., Shamseer, L., Clarke, M., Ghersi, D., Liberati, A., Peticrew, M., Shekelle, P., Stewart, L. A., \& PRISMA-P Group. (2015). Preferred reporting items for systematic review and meta-analysis protocols (PRISMA-P) Statement. Systematic Reviews, 4(1), 1. https://doi.org/10.1186/2046-4053-4-1.

Neves, C. M., Filgueiras, J. F., Fortes, L.S, \& Ferreira, M. E. C. (2013). Comportamentos alimentares em ginastas de elite: associação com o perfeccionismo e o estado de humor. Revista Da Educacao Fisica, 24(3), 359-369. https://doi.org/10.4025/ reveducfis.v24.3.20850.

Neves, C. M., Filgueiras Meireles, J. F., Berbert de Carvalho, P. H., Schubring, A., Barker-Ruchti, N., \& Caputo Ferreira, M. E. (2017). Body dissatisfaction in women's artistic gymnastics: a longitudinal study of psychosocial indicators. Journal of Sports Sciences, 35(17), 1745-1751. https://doi.org/10.1080/ 02640414.2016 .1235794$.

Neves, C. M., Meireles, J. F. F., Carvalho, P. H. B. D., Almeida, S. S., \& Ferreira, M. E. C. (2016a). Body dissatisfaction among Artistic Gymnastics adolescent athletes and non-athletes. Revista Brasileira de Cineantropometria \& Desempenho Humano, 18(1), 8292. http://dx.doi.org/10.5007/19800037.2016v18n1p82.

Neves, C. M., Meireles, J. F. F., Costa, J. L., Pereira, L. C. R., \& Ferreira, M. E. C. (2016b). Influência da mídia e comportamento alimentar de adolescentes atletas e não atletas de Ginástica Artística. Revista 
Brasileira de Ciência e Movimento, 24(2), 129-137. https://doi.org/10.18511/0103-1716/ rbcm.v24n2p129-137.

Palacios de Espig, V., \& Sánchez, A. (2016). Composición corporal y riesgo de trastorno de conducta alimentaria en atletas de Gimnasia Rítmica del Estado Carabobo. Salus, 20(3), 7-11.

Picard, C. L. (1999). The level of competition as a factor for the development of eating disorders in female collegiate athletes. Journal of Youth and Adolescence, 28(5), 583-594.

Rodríguez, A., Fernández, A., Ruiz de Azua, S., \& Goñi, E. (2005). Deporte, influjos socioculturales y trastornos de la alimentación en escolares de Educación Secundaria Obligatoria,. In CongresoVirtual de Investigación en la Actividad Física y el Deporte,Vitoria: Instituto Vasco de Educación Física.

Roever, L. (2017). Compreendendo os estudos de revisão sistemática. Revista da Sociedade. Brasileira de Clínica Médica, 15(2), 127-30.

Strong, W. B., Malina, R. M., Blimkie, C. J., Daniels, S. R., Dishman, R. K., Gutin, B., Hergenroeder, A. C., Must, A., Nixon, P.A., Pivarnik, J. M., Rowland, T., Trost, S., \& Trudeau, F. (2005). Evidence based physical activity for school-age youth. The Journal of Pediatrics, 146(6), 732-737.https://doi.org/10.1016/ j.jpeds.2005.01.055.

Taboada-Iglesias, Y., Vernetta, M., \& Gutiérrez-Sánchez, A. (2017). Anthropometric profile in different event categories of Acrobatic Gymnastics. Journal of Human Kinetics, 57, 169-179. https://doi.org/10.1515/ hukin-2017-0058.

Taboada-Iglesias, Y., Vernetta-Santana, M., Alonso-
Fernández, D., \& Gutierrez-Sánchez, A. (2019). Especificidad antropométrica y nivel de participación en gimnasia acrobática en función del sexo. International Journal of Morphology, 37(4), 1534-1540. http: / / dx.doi.org/10.4067/S071795022019000401534.

Trejo, P. M., Castro, D., Facio, A., Mollinedo, F.E., \& Valdez, G. (2010). Insatisfacción con la imagen corporal asociada al índice de masa corporal en adolescentes. Revista Cubana de Enfermería, 26(3), 144-154.

Vaquero-Cristobal, R., Alacid, F., Muyor, J. M. y LopezMinarro, P. A. (2013). Body image; literature review. Nutricion Hospitalaria, 28(1), 27-35. https: / / doi.org/ 10.3305/nh.2013.28.1.6016.

Verdugo, G., HernándezArmas, E., Baños, R., MoncadaJiménez, J., \& Rentería, I. (2020). Distorsión de la imagen corporal y trastornos alimentarios en adolescentes gimnastas respecto a un grupo control de adolescentes no gimnastas con un imc similar. Retos, 41(37), 297-302. https://doi.org/10.47197/ retos.v37i37.67090.

Vieira, J. L. L., Amorim, H. Z., Vieira, L. F., Amorim, A. C., \& Rocha, P. G. M. da. (2009a). Distúrbios de atitudes alimentares e distorção da imagem corporal no contexto competitivo da Ginástica Rítmica. Revista Brasileira de Medicina Do Esporte, 15(6), 410414. https: / / doi.org/10.1590/s151786922009000700001.

Vieira, J. L. L., Vieira, L. F., Amorim, H. Z., Amorim, A. C., \& Rocha, P. G. M. da. (2009b). Distúrbios de atitudes alimentares e sua relação com o crescimento físico de atletas paranaenses de Ginástica Rítmica. Motriz: Revista da educaçao física, 15(3), 552-561.

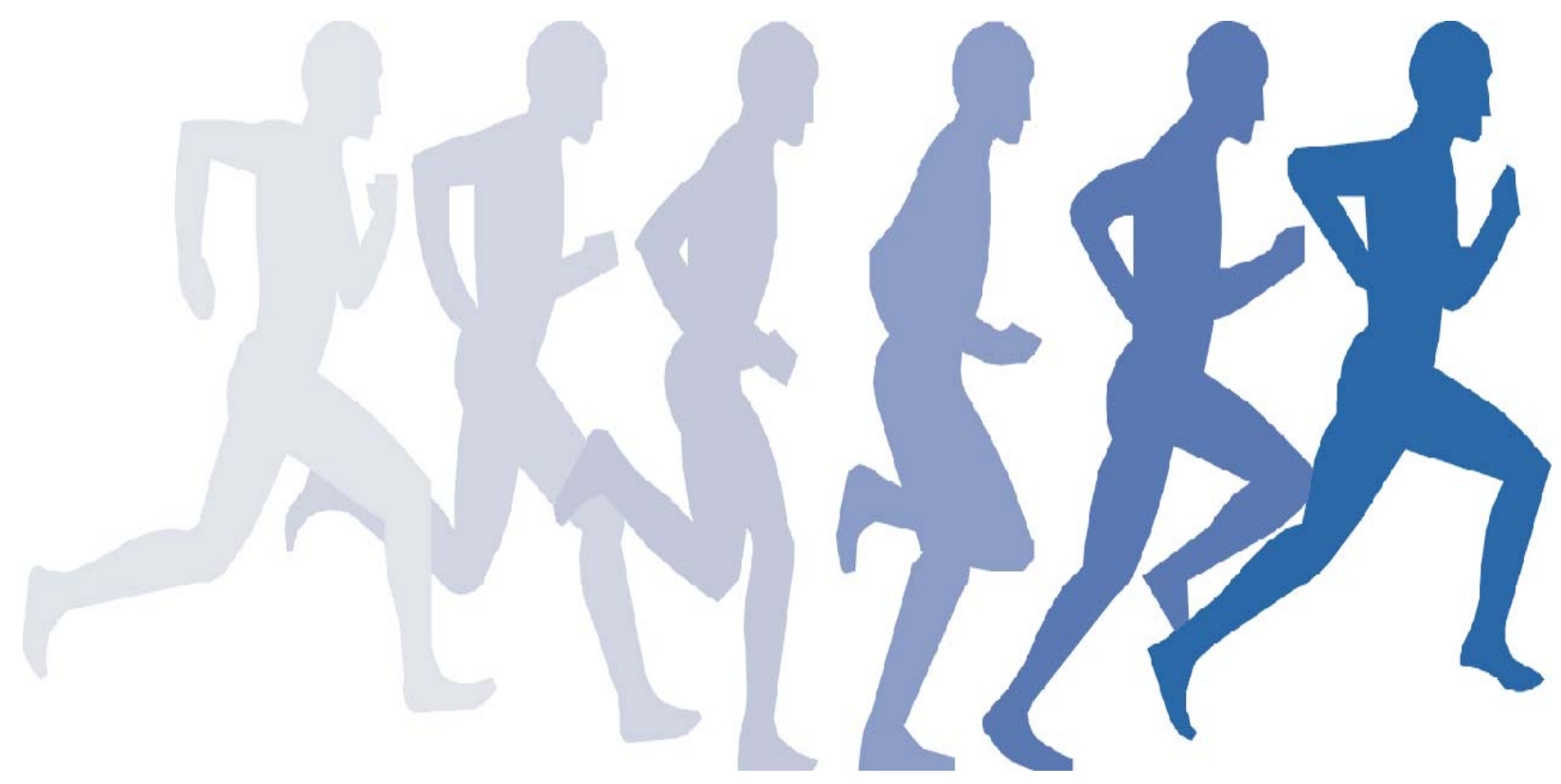

